\title{
Editorial: Advances in Statistical Ecology: New Methods and Software
}

\author{
Gilles Guillot ${ }^{1,2 *}$, Ali Arab $^{3}$, Janine Bärbel Illian ${ }^{4}$ and Stéphane Dray ${ }^{5}$ \\ ${ }^{1}$ GBG Data Analytics, Lyon, France, ${ }^{2}$ World Health Organization (Switzerland), Geneva, Switzerland, ${ }^{3}$ Department of \\ Mathematics and Statistics, Georgetown University, Washington, DC, United States, ${ }^{4}$ School of Mathematics and Statistics, \\ University of Glasgow, Glasgow, United Kingdom, ${ }^{5}$ Laboratoire de Biométrie et Biologie Evolutive, CNRS UMR 5558, \\ Université Claude Bernard Lyon 1, Villeurbanne, France
}

Keywords: stochastic modeling, hidden markov model, computational statistics, ecological data analysis, decision science, population dynamics, $R$ package

\section{Editorial on the Research Topic}

\section{Advances in Statistical Ecology: New Methods and Software}

Ecological studies aim to quantify and explain biological variation at different organization levels, from genes, to individuals, species or communities. The observed variability originates from both ecological processes and from sampling. To adequately analyze data resulting from ecological studies, complex statistical tools are used and developed that reflect the specific sampling methods common to ecological data. This special issue brings together eight contributions in the field of statistical and computational ecology that discuss a variety of ecological data structures and associated statistical modeling approaches applied in variety of different ecological contexts. These include community ecology and abundance modeling (Bystrova et al.; Chiquet et al.), tree population dynamics (Picard et al.), population dynamics and the analysis of capturerecapture data (Jeyam et al.; Worthington et al.), landscape genetics (Hein et al.), animal behavior modeling (Sacchi and Swallow), and decision making in applied conservation ecology (Nagy-Reis et al.). Insight about those questions is brought through the use of matrix population dynamics models (Nagy-Reis et al.), multivariate (mixed) regression models (Chiquet et al.), tree population dynamics models (Picard et al.), Hidden Markov models (Jeyam et al.; Sacchi and Swallow; Worthington et al.), factorial methods and dimension reduction techniques (Bystrova et al.; Hein et al.). The potential of these methods is illustrated through data on various biological models: Rangifer tarandus caribou (Nagy-Reis et al.), microbial communities from oak leaves (Chiquet et al.), Barents sea fish abundance (Chiquet et al.), bacterial communities of Prim'Holstein dairy cattle (Chiquet et al.), Canada geese (Jeyam et al.), gray seals Halichoerus grypus (Worthington et al.), rain forest tree data (Picard et al.), abundance plant species in the Alps (Bystrova et al.) and harbor porpoise Phocoena phocoena (Sacchi and Swallow). Software tools are made available by three groups of authors as R packages PLNmodels (Chiquet et al.) and Wildlift (Nagy-Reis et al.) or free-format R code through github (Sacchi and Swallow).

Despite the diversity of statistical methods, topics and ecological systems, a common theme in the different contributions is the move away from the mere description of patterns in ecological data per se toward the modeling of ecological processes that generate these patterns. This special issue illustrates how this development has been facilitated by the recent development of advanced computational statistical methods and techniques such as parallel MCMC or variational methods. These methods enable the fitting of statistical models, which were unthinkable in the field only 15 years ago. While this selection of papers has certainly been biased toward our own research interests, from a more general perspective highlights 
the wide-ranging contribution the $\mathrm{R}$ language and the broader $\mathrm{R}$ ecosystem have made to both theoretical and applied biosciences.

\section{AUTHOR CONTRIBUTIONS}

All authors listed have made a substantial, direct, and intellectual contribution to the work and approved it for publication.

Conflict of Interest: GG was employed by the company GBG Data Analytics.

The remaining authors declare that the research was conducted in the absence of any commercial or financial relationships that could be construed as a potential conflict of interest.
Publisher's Note: All claims expressed in this article are solely those of the authors and do not necessarily represent those of their affiliated organizations, or those of the publisher, the editors and the reviewers. Any product that may be evaluated in this article, or claim that may be made by its manufacturer, is not guaranteed or endorsed by the publisher.

Copyright (C) 2022 Guillot, Arab, Illian and Dray. This is an open-access article distributed under the terms of the Creative Commons Attribution License (CC BY). The use, distribution or reproduction in other forums is permitted, provided the original author(s) and the copyright owner(s) are credited and that the original publication in this journal is cited, in accordance with accepted academic practice. No use, distribution or reproduction is permitted which does not comply with these terms. 